\title{
Mit Herz und Schenkel
}

\section{Prolog}

Jährlich werden fast 200 Tonnen tiefgefrorene Hinterbeine und Lebendfrösche importiert. Die Vorschriften des Bundesamtes für Veterinärwesen verlangen, dass die Tiere «unter tierschützerisch und hygienisch einwandfreien Bedingungen gehalten und getötet werden». Punkt 10 bestimmt für Packung und Transport der lebenden Ware, dass nicht mehr als zwei Lagen übereinanderzuschichten sind. Dass in Bangladesch, Indien und Indonesien die Tiere beinahe ausgerottet und damit Mückenplagen verursacht wurden, fällt nicht in unsere Zuständigkeit. Vor allem die Romandie liebt Schenkel. Kaum ein Lokal der gehobenen Gastronomie in Genf will ohne diesen Leckerbissen auskommen.

\section{Akt}

Der Professor vom «Laboratoire de pharmacologie» der Universität Genf hat das fünfseitige Gesuchformular A «Demande d'autorisation/ Annonce d'expériences sur animaux» vollständig ausgefüllt an das Kantonale Veterinäramt geschickt. Er beantragt für das Wintersemester einen Frosch pro Student und einen weiteren pro Gruppe, total 52 Tiere. Sie sollen abgekühlt (narcose dans la glace) und durch eine Fachperson dezerebriert werden, bevor das freipräparierte Herz als isolierter Muskel für pharmakologische und elektrophysiologische Experimente dient. Die Firma «Escargots du Mont d'Or» liefert zuverlässig «cuisses de grenouilles fraîches et surgelées» oder «grenouilles vivantes», das Kilogramm für 17 Franken. Vier Monate später stellt das Amt zusätzliche Fragen: Verfügen alle beteiligten Personen über einen Ausbildungsnachweis, und wäre ein Frosch für drei Studenten nicht auch genug? Beigelegt der Hinweis auf interaktive Simulationsprogramme als Alternative: «From guinea pig to computer mouse». Das umgehend erneuerte Gesuch reduziert die Anzahl der Frösche und betont, dass die Tiere keine Qualen erdulden würden. Als Antwort folgt die Stellungnahme eines Mitgliedes der Subkommission zur Überwachung von Tierversuchen. Dieses schlägt vor, an Stelle der realen Herzmuskeln Kunststoffabgüsse zu gebrauchen, und bezeichnet das Vorhaben als schockierend und sowohl wissenschaftlich wie pädagogisch äusserst zweifelhaft. Eine weitere Beilage zitiert «Anima-L», eine Studentenbewegung in Lausanne, die aus Gewissensgründen Tiersektionen in der Biologie und Medizin ablehnt und vollständig auf virtuelle Physiologie setzt. Der Professor bleibt hartnäckig, doch sein Gesuch wird erneut abgewiesen, beiliegend noch einmal der Aufruf der «Association Anima-L». Für das nächste Semester wird eine Zusammenarbeit mit der Medizinischen Fakultät empfohlen.

\section{Akt}

Der Rechtsdienst der Universität nimmt sich der Sache an. Er informiert ausführlich den Gesuchsteller über das komplizierte Prozedere eines Rekursverfahrens, über die unbedingt einzuhaltenden Fristen, den ungewissen Ausgang und die $\mathrm{zu}$ vermutenden Kosten. Einem weiteren Briefwechsel folgt ein neues Gesuch Formular A. 
Die Bewilligung wird, dieses Mal postwendend, verweigert; das geplante Experiment sei für die professionelle Zukunft der Studenten völlig belanglos. Nun bestätigt das Verwaltungsgericht des Kantons Genf die Rekurseingabe. Es folgt eine Stellungnahme der Rekurskommission des Eidgenössischen Volkswirtschaftsdepartements mit einer Präzisierung der Rechtslage, die unter anderem festhält, dass Autopsien von Tieren für Lehrzwecke keiner Bewilligung bedürfen, wenn diese fachgerecht getötet wurden. Die Argumente des Antragstellers werden in allen Einzelheiten bestätigt. Das Verwaltungsgericht verweigert dennoch eine provisorische Bewilligung und verweist auf den ausstehenden Gerichtsentscheid, gestützt auf die ausführlichen Berichte beider Parteien, inklusive Protokoll der Subkommission für Tierversuche. Am 1. November 2005, 17 Monate nach Einreichen des ersten Gesuches, wird vom Verwaltungsgericht Genf zugunsten der Universität entschieden. Das Gericht wun- dert sich («Il est pour le moins piquant que ...»), dass das «Office vétérinaire cantonal» die Bewilligung noch $\mathrm{zu}$ einem Zeitpunkt verweigerte, als ihm bewusst war, dass es in diesem Fall gar keine Bewilligung brauchte.

\section{Epilog}

Und die Moral von der Geschichte: Frosch ist nicht gleich Frosch. Den Gourmet kümmert es nicht, was die Viecher auf seinem Teller für eine Geschichte haben, doch was für ein Labor gut ist, weiss er allemal. Eine amerikanische Literaturübersicht bestätigt unsere selektive Wahrnehmung konsumierter Lebewesen: Ein Drittel der Kritik betrifft die 95 Prozent, die in unserer Küche enden, zwei Drittel konzentrieren sich auf die 0,3 Prozent aus Forschung und Lehre.

Wir sind also 633mal weniger kritisch gegenüber eigenem Fleischverzehr als bezüglich Verwendung in wissenschaftlichen Experimenten. 